\title{
Bayesian Wavelet Shrinkage With Edge Detection for SAR Image Despeckling
}

\author{
Min Dai, Student Member, IEEE, Cheng Peng, Andrew K. Chan, Fellow, IEEE, and Dmitri Loguinov, Member, IEEE
}

\begin{abstract}
In this paper, we present a wavelet-based despeckling method for synthetic aperture radar images and derive a Bayesian wavelet shrinkage factor to estimate noise-free wavelet coefficients. To preserve edges during despeckling, we apply a modified ratio edge detector to the original image and use the obtained edge information in our despeckling framework. Experimental results demonstrate that our method compares favorably to several other despeckling methods on test images.
\end{abstract}

Index Terms-Minimum mean square error (MMSE) estimation, ratio edge detector, stationary wavelet transform (SWT), wavelet shrinkage.

\section{INTRODUCTION}

$\mathbf{S}$ YNTHETIC aperture radar (SAR) images provide useful information for many applications, such as oil slick pollution monitoring and internal coastal wave detection [1]. However, many imaging systems produce images with speckles, which reduce the detectability of targets and impede further investigation of SAR images. Thus, speckle reduction (despeckling) has become an important issue in SAR image processing. Many algorithms have been developed for despeckling, including the Lee filter [2], the Frost filter [3], the Gamma MAP filter [4], and their variations [1], [5]. These standard filters usually perform well in despeckling; however, they typically exhibit limitations in preserving sharp features and/or details of the original image [6].

To overcome these disadvantages, wavelet-based techniques have been widely utilized in SAR image processing [6]-[9]. Wavelet denoising methods usually apply hard thresholding or soft thresholding (i.e., shrinkage factor) to the detailed wavelet coefficients of noisy images [10]. Since speckles in SAR images are multiplicative in nature, many wavelet-based despeckling approaches apply the log-transform to SAR images to statistically convert the multiplicative noise to additive noise before performing further wavelet denoising [6], [9], [11]. After wavelet denoising, an exponential operation is employed to convert the log-transformed images back to the nonlogarithmic format [9]. However, the mean of log-transformed speckle

Manuscript received January 31, 2003; revised April 13, 2004.

M. Dai and A. K. Chan are with the Electrical Engineering Department, Texas A\&M University, College Station, TX 77843 USA (e-mail: min@ee.tamu.edu; akchan@ee.tamu.edu).

C. Peng was with the Electrical Engineering Department, Texas A\&M University, College Station, TX 77843 USA. He is now with Texas Instruments, Stafford, TX 77477 USA (e-mail: c-peng2@ ti.com).

D. Loguinov is with the Computer Science Department, Texas A\&M University, College Station, TX 77843 USA (e-mail: dmitri@cs.tamu.edu).

Digital Object Identifier 10.1109/TGRS.2004.831231 noise does not equal to zero [12] and thus requires correction to avoid extra distortion in the restored image.

There are also several wavelet-based techniques that avoid the log-transform [7], [8], [13], [14]. Sveinsson et al. [13] directly apply the enhanced Lee filter in the discrete wavelet transform (DWT) domain to reduce the presence of speckles. Xie et al. [14] propose a low-complexity wavelet denoising process based on the minimum mean square error (MMSE) estimation. Although DWT is widely applied in the area of signal denoising and image compression, the downsampling operation in DWT results in a time-variant translation and has difficulties preserving original image discontinuities in the wavelet domain [8].

Another approach involves the stationary wavelet transform (SWT), which is a time-invariant transform commonly used for denoising purposes [15], [16]. In [8], Foucher et al. use the Pearson distribution to model the probability density function (pdf) of SWT wavelet coefficients and reconstruct the despeckled image using the maximum a posteriori (MAP) criterion. Although this algorithm has sound performance, the high computational complexity of the Pearson distribution makes this approach less appealing in practice. Similar to [14], Argenti et al. [7] apply a local linear MMSE estimator in the wavelet domain. The major differences between these two methods are the wavelet transform applied (stationary [7] or nonstationary [14]) and the specific estimation related to the unknown noise-free signal in the wavelet domain [8].

In this paper, we propose an efficient SWT despeckling method that preserves edges of the original image. We avoid the log-transform and derive a novel wavelet shrinkage factor based on the mixture-Gaussian distribution model of wavelet coefficients. Since edge information is very useful in preserving the edges during despeckling, we modify the ratio edge detector in [17] to obtain necessary edge information with lower computational complexity. Several typical images are used to evaluate the despeckling performance of our method along with some standard filters and several other wavelet-based methods [9], [14].

This paper is organized as follows. In Section II, we describe statistical properties of SAR images, the two-dimensional (2-D) SWT algorithm, and a statistical model of wavelet coefficients. In Section III, we give a brief review of related work on shrinkage factors, derive a wavelet shrinkage factor, and provide a mechanism to estimate noise-free wavelet coefficients. In Section IV, a modified ratio edge detector is introduced. Section V provides a description of our algorithm and shows experimental results. Finally, a short conclusion is given in Section VI. 


\section{Statistical Models For SAR IMAGES AND CORRESPONDING WAVELET COEFFICIENTS}

\section{A. Statistical Models for SAR Images}

In this paper, we only consider the statistical properties of amplitude and intensity SAR images. Let $X$ be the observed signal (intensity or amplitude), $Y$ be the noise-free signal, and $F$ be the speckle noise. Since speckle noise $F$ is multiplicative in nature, the observed signal can be expressed as $X=Y F$. Next, recall that the observed intensity $I$ of an $L$-look image has the conditional pdf given by [1]

$$
p_{I \mid Y}(x \mid y)=\frac{1}{\Gamma(L)}\left(\frac{L}{y}\right)^{L} x^{L-1} e^{-L x / y}
$$

where $x$ represents an observed intensity value, $y$ is the corresponding actual intensity value, and $\Gamma(\cdot)$ is the gamma function. Amplitude $A$, which is the square root of intensity $I$, is distributed with the following pdf [1]:

$$
p_{A \mid Y}(x \mid y)=\frac{2}{\Gamma(L)}\left(\frac{L}{y}\right)^{L} x^{2 L-1} e^{-L x^{2} / y}
$$

Note that using $L=1$ in (1) and (2) produces the distribution of monolook intensity and amplitude, which are exponential and Rayleigh distributions, respectively.

In an $L$-look intensity image, speckle noise $F$ is a unit-mean gamma-distributed variable of order $L$ and its pdf is given by [8], [14]

$$
p_{F}(f)=\frac{L^{L} f^{L-1} e^{-L f}}{\Gamma(L)}, \quad f \geq 0
$$

where $f$ is a speckle noise random variable. Notice that for $L=$ 1 , (3) simplifies to a unit-mean exponential distribution. In an $L$-look amplitude image, the distribution of speckle noise $F$ is obtained numerically, but its mean equals one and its variance equals $(4 / \pi-1) / L$. For detailed information, the readers are referred to [1] and [18].

\section{B. Stationary Wavelet Transform}

The main strength of SWT is its time-invariance property [15], which is useful in many applications (e.g., breakdownpoints detection and denoising [19]). The SWT algorithm is slightly different from that of DWT. Fig. 1 shows the 2-D SWT, where $H_{j}$ and $L_{j}$ are the highpass and lowpass filters at level $j$, respectively. Also, note in the figure that $L L_{0}$ is the original image and that the output $L L_{j}$ of each level $j$ is fed into the input of the next level $j+1$. As shown in Fig. 1, filters $H_{j}$ and $L_{j}$ are upsampled by two from filters $H_{j-1}$ and $L_{j-1}(j>1)$. Since SWT does not include downsampling operations, it is a redundant transform.

\section{Statistical Models of Wavelet Coefficients}

Wavelet coefficients of SAR images typically exhibit strong non-Gaussian statistics [8], [12]. Chang et al. [20] model wavelet coefficients with a generalized Gaussian distribution (GGD), which matches well histograms of typical SAR images. However, GGD is not analytically easy to work with due to its

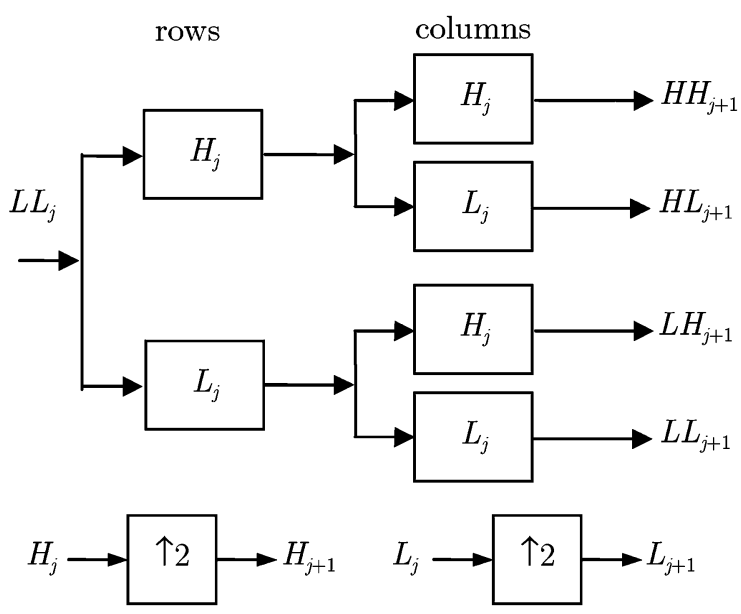

Fig. 1. Two-dimensional SWT algorithm.

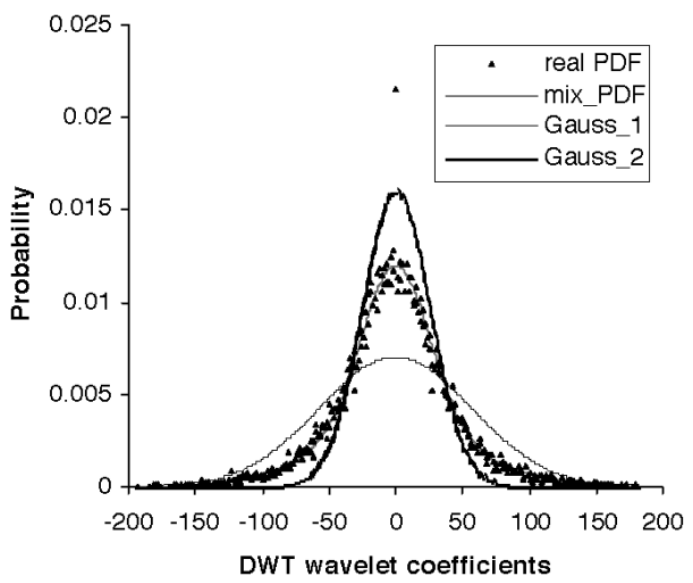

Fig. 2. Histogram of DWT wavelet coefficients from Bedfordshire and the mixture-Gaussian model.

complex structure. Among alternative methods, a mixture density of two zero-mean Gaussian distributions has been proposed due to its relatively simple form and high accuracy in modeling the distribution of wavelet coefficients [21]. Assuming that $W_{X}$ represents the noisy wavelet coefficients, its mixture pdf is given by

$$
p_{W_{X}}\left(w_{X}\right)=\sum_{k=0,1} p(S=k) p\left(w_{X} \mid S=k\right)
$$

where $p\left(w_{X} \mid S=k\right)$ is a zero-mean Gaussian distribution and $S=0$ or 1 represents each Gaussian component in the mixture distribution.

We demonstrate two typical examples in Figs. 2 and 3 to show the performance of the mixture-Gaussian model in matching the distribution of DWT and SWT wavelet coefficients of a real SAR image (taken in Bedfordshire, southeast England). In the figures, the mixture Gaussian distribution is labeled as "mix_PDF" and the two pure Gaussian components are labeled as "Gauss_1" and "Gauss_2," respectively. As the figures show, the mixture-Gaussian model follows the actual histograms very well (note that a small discrepancy near zero does not reduce the effectiveness of the model). Based on the mixture-Gaussian model, we build a denoising method in the following section. 


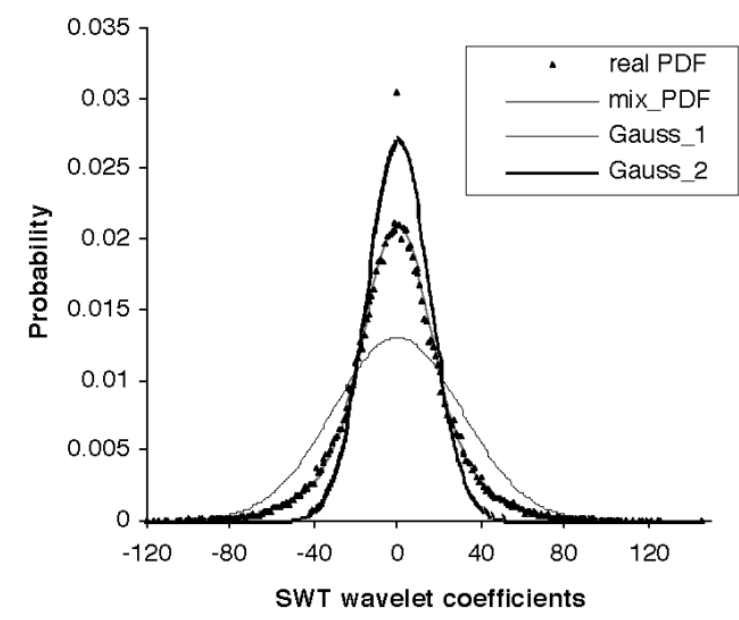

Fig. 3. Histogram of SWT wavelet coefficients from Bedfordshire and the mixture-Gaussian model.

\section{BAYESIAN WAVELET ESTIMATE}

In this section, we derive a novel wavelet shrinkage factor and use it to estimate noise-free wavelet coefficients. Recall that in the wavelet domain, multiplicative speckle noise can be converted into additive noise without the use of the log-transform [7], [8], [14]

$$
\begin{aligned}
W_{X} & =W[X]=W[Y F] \\
& =W[Y]+W[Y(F-1)] \\
& =W_{Y}+W_{B}
\end{aligned}
$$

where $W_{B}$ is an additive signal-dependent noise equal to $W[Y(F-1)]$. Shrinkage estimate of the noise-free wavelet coefficient $W_{Y}$ is $\hat{W}_{Y}=\eta W_{X}$, where $\eta$ is the shrinkage factor. The shrinkage factor is often clipped at zero and the estimated wavelet coefficients can be written as $\hat{W}_{Y}=\max (0, \eta) W_{X}$ [7], [14]. Based on the MMSE criteria, the optimal shrinkage factor $\eta^{\prime}$ is obtained by minimizing the mean square error between $\hat{W}_{Y}$ and $W_{Y}$

$$
\eta^{\prime}=\arg \min _{\eta} E\left[\left(\hat{W}_{Y}-W_{Y}\right)^{2}\right]
$$

which has the MMSE solution in the form of

$$
\eta^{\prime}=\frac{E\left[W_{X}^{2}\right]-E\left[W_{X} W_{B}\right]}{E\left[W_{X}^{2}\right]}
$$

To calculate $\eta^{\prime}$, it is necessary to estimate all unknown parameters in (7) [7], [14]. In the rest of this section, we briefly overview several methods of calculating the shrinkage factor and derive a novel estimator of $\eta^{\prime}$.

\section{A. Related Work}

In (7), it is difficult to precisely estimate $E\left[W_{X} W_{B}\right]$ due to the dependence of noise $W_{B}$ upon $W_{X}$. Xie et al. [14] assume that the wavelet basis functions are short enough and then compute $E\left[W_{X} W_{B}\right]$ as

$$
E\left[W_{X} W_{B}\right]=\sigma_{W_{B}}^{2} \sum_{p=0}^{N-1} \sum_{q=0}^{N-1} h_{p}^{2} \hat{h}_{q}^{2} Y_{p, q}^{2}
$$

where $h$ and $\hat{h}$ denote the wavelet filters applied to the row and column of the original image, respectively, $\sigma_{W_{B}}^{2}$ is the variance of noise $W_{B}$, and unknown noise-free signal $Y$ is estimated from the coefficients in the LL subband.

Different from [14], Argenti et al. [7] apply a local linear MMSE estimator to obtain $W_{Y}$

$$
\begin{aligned}
\hat{W}_{Y} & =\max \left(0, \frac{E\left[W_{Y}^{2}\right]}{E\left[W_{X}^{2}\right]}\right) W_{X} \\
& =\max \left(0, \frac{E\left[W_{X}^{2}\right]-E\left[W_{B}^{2}\right]}{E\left[W_{X}^{2}\right]}\right) W_{X} .
\end{aligned}
$$

For a random pixel $n, E\left[W_{B}^{2}\right]$ at decomposition level $j$ is estimated by [7]

$$
\begin{aligned}
E\left[W_{B}^{2}\right]= & \frac{\sigma_{u}^{2}}{1+\sigma_{u}^{2}} \sum_{i} H_{j}(i)^{2} E\left[X(n-i)^{2}\right] \\
& +\sum_{i} \sum_{k, k \neq i} H_{j}(i) H_{j}(k) \\
& \cdot E[X(n-i)] E[X(n-k)] R_{u u}(i-k)
\end{aligned}
$$

where $H_{j}$ is the highpass filter at level $j$, random process $u$ equals $F-1$, and $R_{u u}$ is the autocorrelation function of noise $u$. Notice that (10) applies only to one-dimensional signals and that 2-D images require much more filtering and multiplications. Furthermore, (10) involves the estimation of unknown parameter $R_{u u}$. Both of these observations show that the computation of (9) is rather expensive.

\section{B. Novel Shrinkage Factor}

To avoid high computational complexity, we derive the shrinkage factor from another perspective.

Lemma 1: Based on a mixture-Gaussian model of wavelet coefficients, the MMSE estimate of noise-free wavelet coefficients $\hat{W}_{Y}$ is

$$
\hat{W}_{Y}=\sum_{k=0,1} p\left(S=k \mid w_{X}\right) \frac{\sigma_{W_{X}}^{2}-\sigma_{W_{B}}^{2}}{\sigma_{W_{X}}^{2}} W_{X}
$$

where $\sigma_{W_{X}}^{2}$ is the variance of the noisy wavelet coefficients $W_{X}$ in the corresponding state $S$ and $p\left(S=k \mid w_{X}\right)$ is calculated based on the Bayes rule

$$
p\left(S=k \mid w_{X}\right)=\frac{p\left(w_{X} \mid S=k\right) p(S=k)}{p\left(w_{X}\right)} .
$$

Proof: Recall that $W_{X}=W_{Y}+W_{B}$ in (5). After replacing $W_{X}$ with $W_{Y}+W_{B}$ in $E\left[W_{X} W_{B}\right]$, we have

$$
E\left[W_{X} W_{B}\right]=E\left[W_{Y} W_{B}\right]+E\left[W_{B}^{2}\right] .
$$

Since the speckle-noise random variable $F$ is usually normalized

$$
\begin{aligned}
E[F] & =1 \\
E[F-1] & =0 .
\end{aligned}
$$

Thus, due to the highpass nature of wavelet functions, we have $E\left[W_{B}\right]=0$. Furthermore, because of the zero-mean mixture- 
Gaussian distribution model of $W_{X}$, its expectation $E\left[W_{X}\right]$ is also zero. Therefore, we obtain

$$
\begin{aligned}
E\left[W_{X}^{2}\right] & =\sigma_{W_{X}}^{2} \\
E\left[W_{B}^{2}\right] & =\sigma_{W_{B}}^{2} .
\end{aligned}
$$

Since $W_{Y}$ and $W_{B}$ are statistically independent

$$
E\left[W_{Y} W_{B}\right]=E\left[W_{Y}\right] E\left[W_{B}\right]=0
$$

and $E\left[W_{X} W_{B}\right]$ becomes

$$
E\left[W_{X} W_{B}\right]=0+E\left[W_{B}^{2}\right]=\sigma_{W_{B}}^{2}
$$

Combining (16), (17), and (19), the new shrinkage factor $\eta^{\prime}$ is given by

$$
\eta^{\prime}=\frac{E\left[W_{X}^{2}\right]-E\left[W_{X} W_{B}\right]}{E\left[W_{X}^{2}\right]}=\frac{\sigma_{W_{X}}^{2}-\sigma_{W_{B}}^{2}}{\sigma_{W_{X}}^{2}}
$$

which represents both (7) and (9) based on a mixture-Gaussian model of wavelet coefficients. Combining (20) with (4), we obtain an estimate of noise-free wavelet coefficients $\hat{W}_{Y}$, as shown in (11).

The unknown probabilities in (12) are calculated with the expectation-maximization (EM) algorithm [22].

\section{Parameter Estimation}

Notice that in (11), all parameters except $\sigma_{W_{B}}^{2}$ can be easily estimated from the observed noisy signal. In the following exposition, we derive $\sigma_{W_{B}}^{2}$ using the results of [8].

Lemma 2: After the SWT transform, the variance of noise in the wavelet domain $\sigma_{W_{B}}^{2}$ equals

$$
\sigma_{W_{B}}^{2}=\frac{\Psi_{j} \mu_{X}^{2}+\sigma_{W_{X}}^{2}}{1+C_{F}^{2}} C_{F}^{2}
$$

where $\mu_{X}=E[X]$, the normalized standard deviation of noise $C_{F}$ equals $\sqrt{1 / L}$ for intensity images and $\sqrt{(4 / \pi-1) / L}$ for amplitude images $(L \geq 1)$, and parameter $\Psi_{j}$ is defined as

$$
\Psi_{j}=\left(\sum_{k}\left(h_{k}\right)^{2}\right)^{2}\left(\sum_{l}\left(g_{l}\right)^{2}\right)^{2(j-1)}
$$

where $h$ and $g$ are the highpass and lowpass filters at decomposition level $j$, respectively.

Proof: Based on the statistical properties of SWT transform and SAR images, Foucher et al. [8] estimate $\sigma_{W_{B}}^{2}$ as

$$
\sigma_{W_{B}}^{2}=\Psi_{j} \mu_{Y}^{2} C_{F}^{2}\left(1+C_{Y}^{2}\right)
$$

where $\mu_{Y}=E[Y]$ and $C_{Y}^{2}$ is

$$
C_{Y}^{2}=\frac{C_{W_{X}}^{2}-\Psi_{j} C_{F}^{2}}{\Psi_{j}\left(1+C_{F}^{2}\right)} .
$$

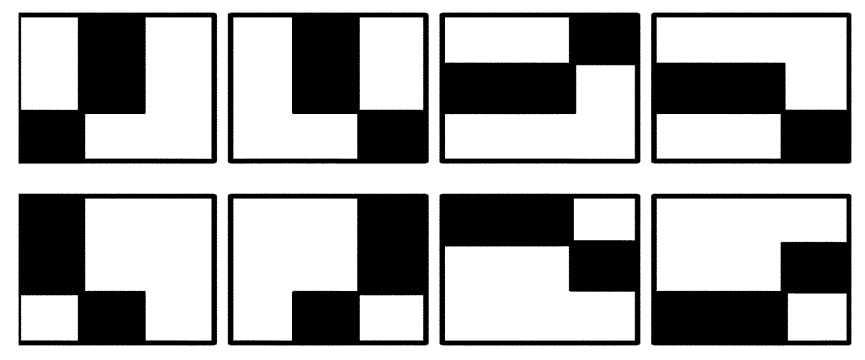

Fig. 4. Eight flat-slope directions.

The normalized standard deviation of noisy wavelet coefficients $C_{W_{X}}$ is

$$
C_{W_{X}}=\frac{\sigma_{W_{X}}}{\mu_{X}}
$$

Recall that $E[X]=E[Y]$ with normalized speckle noise $F$. Inserting (24) and (25) into (23), we obtain

$$
\begin{aligned}
\sigma_{W_{B}}^{2} & =\Psi_{j} \mu_{Y}^{2} C_{F}^{2} \frac{\Psi_{j}\left(1+C_{F}^{2}\right)+C_{W_{X}}^{2}-\Psi_{j} C_{F}^{2}}{\Psi_{j}\left(1+C_{F}^{2}\right)} \\
& =\mu_{X}^{2} C_{F}^{2} \frac{\Psi_{j}+\frac{\sigma_{W_{X}}^{2}}{\mu_{X}^{2}}}{\left(1+C_{F}^{2}\right)} \\
& =\frac{C_{F}^{2}\left(\Psi_{j} \mu_{X}^{2}+\sigma_{W_{X}}^{2}\right)}{\left(1+C_{F}^{2}\right)}
\end{aligned}
$$

which is the same as (21).

\section{RATIO EDGE DETECTOR}

Since edge information is important in preserving image details during despeckling [7], [8], our algorithm employs a modified edge detector whose details are presented in this section. Recall that Touzi et al. [17] propose a ratio edge detector, which operates as following. Assuming $P_{1}$ and $P_{2}$ are the arithmetic means of pixel values of the two nonoverlapping neighborhoods on the opposite sides of pixel $i$, the ratio detector $r$ equals

$$
r= \begin{cases}R, & R \leq 1 \\ R^{-1}, & R>1\end{cases}
$$

where $R$ is $P_{1} / P_{2}$. The authors of [17] apply a moving window to each pixel, compute ratio $r$ in four directions (i.e, horizontal, vertical, and two diagonal), and then choose the best edge direction according to certain thresholds. To obtain locally optimal detection, the window size and thresholds are adjusted adaptively for each pixel, which dramatically increases the computational complexity of the method.

To achieve efficient edge detection with low computational cost, we use a fixed-size window and a uniform threshold in each test image, but add more detection directions. Besides the four directions that already exist in the original edge detector, we add eight flat-slope directions, which are shown in Fig. 4.

Due to high correlation between the conditional probability $p\left(r \mid P_{1} / P_{2}\right)$ and edge directions, we choose the direction with 


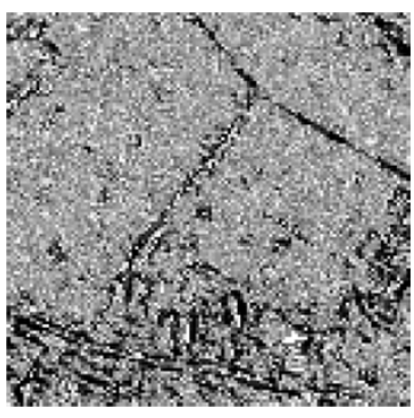

(a)

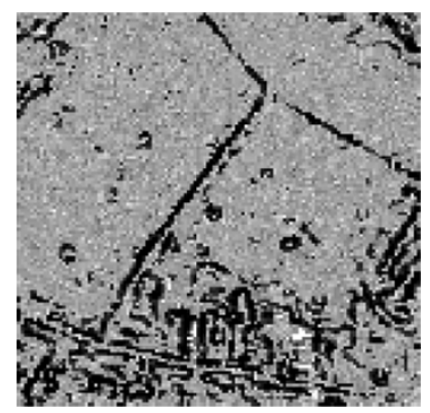

(b)
Fig. 5. With a uniform threshold and a fixed window size, the result of (a) the original edge detector [17] and (b) that of our edge detector.

the highest conditional probability as the most likely edge direction. For an $L$-look SAR image, $p\left(r \mid P_{1} / P_{2}\right)$ is given by [17]

$$
\begin{aligned}
p\left(r \mid \frac{P_{1}}{P_{2}}\right)=\frac{n r^{n M-1} \Gamma(2 M)}{\Gamma(M)^{2}} \\
\cdot\left[\frac{\left(\frac{P_{1}}{P_{2}}\right)^{M}}{\left(r^{n}+\frac{P_{1}}{P_{2}}\right)^{2 M}}+\frac{\left(\frac{P_{2}}{P_{1}}\right)^{M}}{\left(r^{n}+\frac{P_{2}}{P_{1}}\right)^{2 M}}\right]
\end{aligned}
$$

where $n$ equals 1 for intensity images and 2 for amplitude images, $d$ is the width of the window, and $M=d L(d-1) / 2$. In Fig. 5, we compare the performance between the modified edge detector and the original edge detector [17] in a $125 \times$ 125 two-look amplitude SAR image using a uniform threshold and a fixed window size. Even though the original edge detector performs well in [17], Fig. 5(a) shows that this method no longer provides pleasing edge detection results in the low computational complexity case (i.e., a uniform threshold and a fixed window size). Fig. 5(b) shows that our edge detector preserves existing edges and produces fewer false edges in the homogeneous areas.

\section{AlgORIthM AND EXPERIMENTAL RESUlts}

In this section, we describe our despeckling framework and show experimental results. Since it is possible for wavelet shrinkage to blur edges, we combine Bayesian wavelet shrinkage described earlier in the paper with the edge detector of the last section. The modified edge detector is applied to noisy SAR images, and two thresholds $\left(T_{0}, T_{1}\right)$ are chosen $\left(T_{0}<T_{1}\right)$ according to the content of the image. If the value of the ratio detector $r$ of a pixel $i$ is lower than $T_{0}$, the pixel is considered to be an edge point, and we assume that $\hat{W}_{Y}$ equals $W_{X}$ at the corresponding decomposition level. If $r$ is higher than $T_{1}$, pixel $i$ is considered to be in a homogeneous neighborhood, and $\hat{W}_{Y}$ is set to zero. Otherwise, $\hat{W}_{Y}$ is computed according to (11). Finally, an inverse SWT is applied to obtain the despeckled image. A fixed-size $3 \times 3$ moving window is applied in our algorithm to estimate the local statistics of the original image and the Haar wavelets are used in all filtering.
TABLE I

ENL FOR THE TEST REGIONS

\begin{tabular}{lccc}
\hline Methods & $\begin{array}{c}\text { Bedfordshire } \\
(L=2)\end{array}$ & $\begin{array}{c}\text { GAC } \\
(L=1)\end{array}$ & $\begin{array}{c}\text { CNES } \\
(L=4)\end{array}$ \\
\hline Original & 1.7531 & 0.9602 & 3.6608 \\
Ours & 11.6007 & 10.1169 & 23.2824 \\
Frost [3] & N/A & 2.5025 & 11.7991 \\
Gamma [4] & 11.9903 & 5.9489 & 31.6857 \\
Lee [5] & 5.1268 & 4.8645 & 19.9440 \\
Non-log SWT shrinkage [7] & 9.4276 & 6.7963 & 22.4557 \\
Log-transformed DWT shrinkage [9] & N/A & 2.1483 & 19.2614 \\
Non-log DWT shrinkage [14] & 8.8833 & 5.1830 & 22.5434 \\
Weibull [24] & N/A & 3.6259 & 7.5303 \\
\hline
\end{tabular}

We evaluate the performance of our algorithm in three images: ${ }^{1}$ a two-look X-band amplitude image (Bedfordshire), a monolook intensity image (GAC), and a four-look synthesized amplitude image (CNES). We use equivalent number of looks (ENL) for comparison purposes. ENL is widely applied to measure the smoothing effects of despeckling methods. It is defined as ENL $\mu^{2} / \sigma^{2}$, where $\mu$ and $\sigma^{2}$ are the mean and the variance of intensity value over a uniform image area [1]. The value of ENL theoretically equals the number of look of an intensity image.

In Table I, we compare the smoothing performance (ENL) between our method and several popular despeckling methods in typical homogeneous areas of three test images. Specifically, Table I includes the results of several shrinkage methods: nonlog-transformed SWT shrinkage [7], log-transformed DWT shrinkage [9], and nonlog-transformed DWT shrinkage [14]. The nonlog-transformed methods [7], [14] use Daubechies-4 wavelets and two-level decomposition ${ }^{2}$ in test images.

The size of a test region is important, since it should be large enough to provide a robust estimation of the value of ENL and to allow the homogeneity hypothesis to hold in that region [23]. In our simulation, we set test region size to $50 \times 50$ pixels according to the texture structure of the test images. The Silogic company and CNES provide results of several methods (i.e., see [3], [9], and [24]), which we show in Table I; however, results of these methods are not available (N/A) for the Bedfordshire image. Also, notice that we convert the test regions of the amplitude-formatted Bedfordshire and CNES images to intensity to calculate the ENL.

Table I demonstrates that our method has good performance in the homogeneous areas for both multilook and monolook images. Table I also shows that the Gamma MAP filter performs better than our method in multilook images in terms of ENL. However, recall that ENL is not a good measure of despeckling performance of a method in heterogeneous areas of an image. Thus, we next discuss the actual despeckled images to show the subjective quality of several methods from Table I.

Since the Bedfordshire image is a real SAR image (the CNES image is synthesized) and has richer characteristics than the GAC image, we display some experimental results of the Bedfordshire image. Fig. 6 is the original image. Fig. 7 shows that the Gamma MAP filter exhibits strong blurring effects. Fig. 8

\footnotetext{
${ }^{1}$ The first image is Bedfordshire in Southeast England. The second one is a target detection image provided by the German Aerospace Center (GAC), and the third one is a synthetic image from the French Space Agency (CNES).

2In these two methods, high decomposition level tends to generate over-smoothed despeckled images.
} 


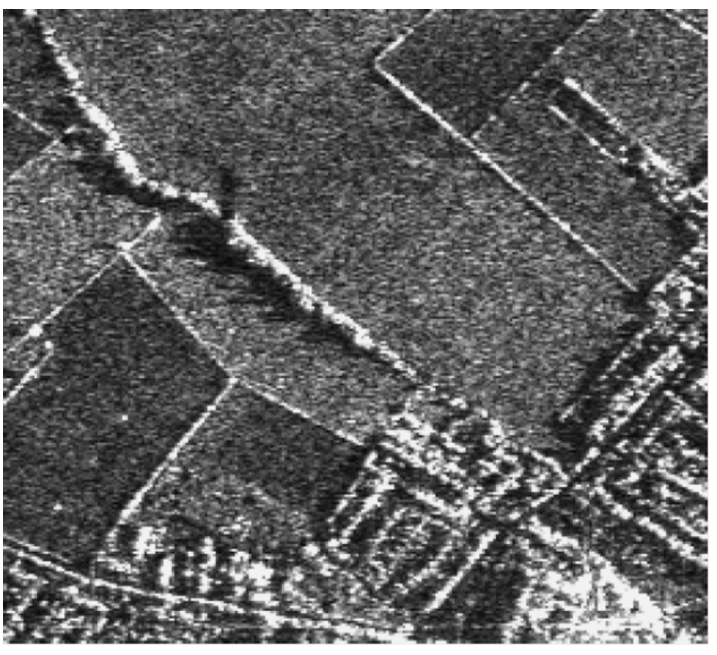

Fig. 6. Original noisy image.

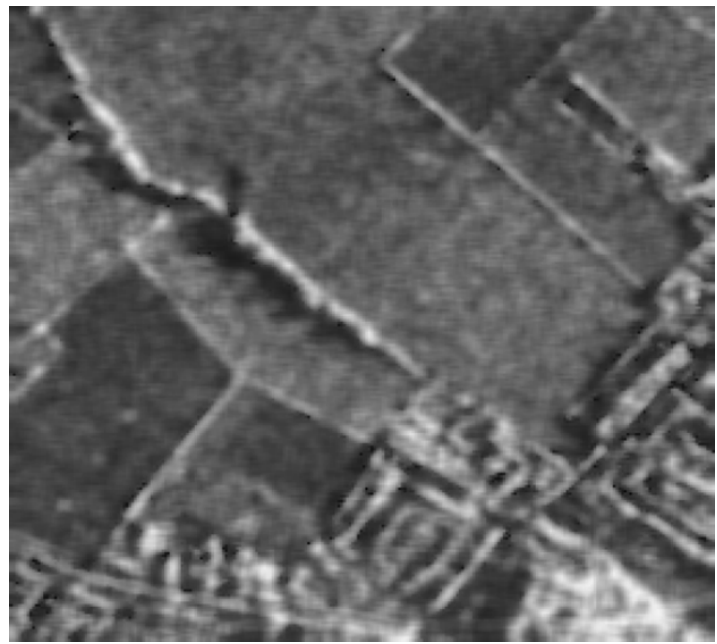

Fig. 7. Result of the Gamma MAP filter $(5 \times 5$ window $)$ [4].

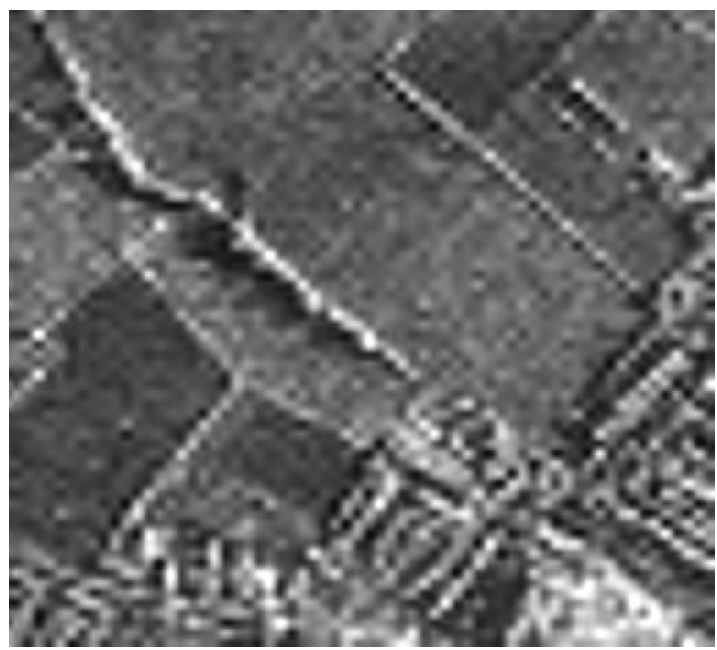

Fig. 8. Result of a nonlog-transformed DWT shrinkage method [14].

indicates that the nonlog-transformed DWT shrinkage method [14] performs better in homogeneous areas than in heterogeneous areas. In Fig. 9, which is the despeckled image of the

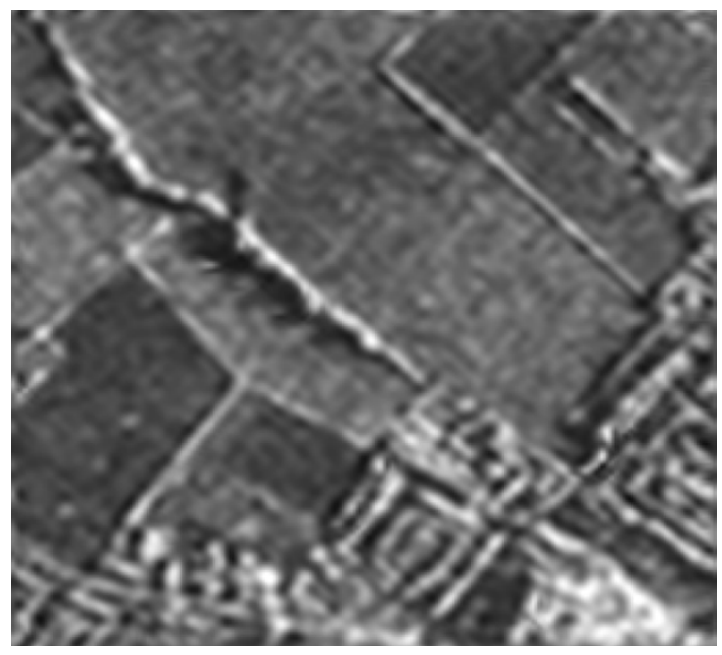

Fig. 9. Result of nonlog-transformed SWT shrinkage method [7].

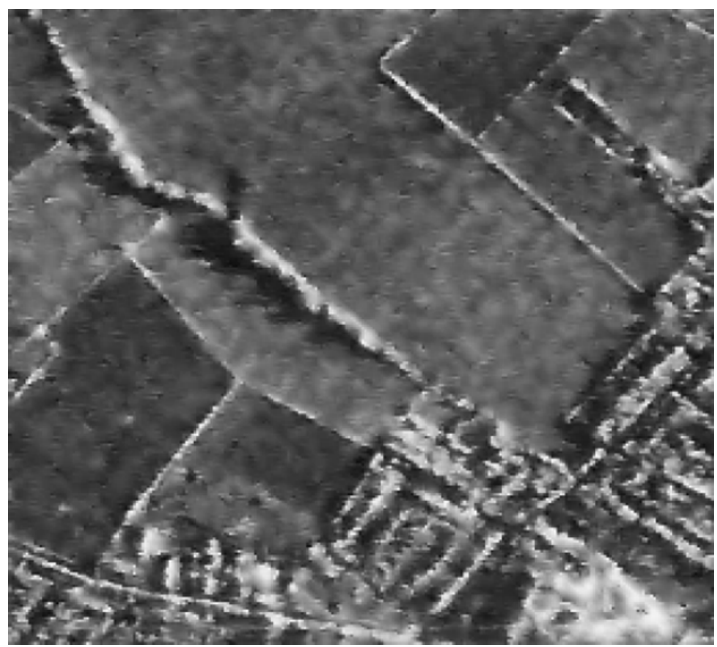

Fig. 10. Result of our method.

nonlog-transformed SWT shrinkage method [7], we still observe speckle noise in homogeneous areas. Fig. 10 shows that our method smoothes the homogeneous area (e.g., the upper part of the image), but also keeps the edges (e.g., the bottom left corner).

\section{CONCLUSION}

In this paper, we presented a wavelet-based despeckling method for SAR images. We derived a novel shrinkage factor and described an efficient ratio edge detector. Experimental results demonstrate that our algorithm has a good despeckling performance in SAR images and typically preserves even the minor edges. In future work, we plan to further improve the performance of this despeckling method by including other techniques, such as the iteration process or simulated annealing.

\section{ACKNOWLEDGMENT}

The authors would like to thank S. Foucher for his generous help and the anonymous reviewers for their constructive comments. 


\section{REFERENCES}

[1] C. Oliver and S. Quegan, Understanding Synthetic Aperture Radar Images. Norwood, MA: Artech House, 1998.

[2] G. Lee, "Refined filtering of image noise using local statistics," Comput. Graph. Image Process., vol. 15, no. 4, 1981.

[3] V. S. Frost, J. A. Stiles, K. S. Shanmugan, and J. C. Holtzman, "A model for radar images and its application to adaptive digital filtering of multiplicative noise," IEEE Trans. Pattern Anal. Machine Intell., vol. PAMI-4, Mar. 1980.

[4] A. Lopes, E. Nezry, R. Touzi, and H. Laur, "Maximum a posteriori filtering and first order texture models in SAR images," in Proc. IGARSS, 1990.

[5] A. Lopes, R. Touzi, and E. Nezry, "Adaptive speckle filters and scene heterogeneity," IEEE Trans. Geosci. Remote Sensing, vol. 28, pp. 992-1000, Nov. 1990.

[6] L. Gagnon and A. Jouan, "Speckle filtering of SAR images-A comparative study between complex-wavelet-based and standard filters," Proc. SPIE, 1997.

[7] F. Argenti and L. Alparone, "Speckle removal from SAR images in the undecimated wavelet domain," IEEE Trans. Geosci. Remote Sensing, vol. 40, pp. 2363-2374, Nov. 2002.

[8] S. Foucher, G. B. Bénié, and J.-M. Boucher, "Multiscale MAP filtering of SAR images," IEEE Trans. Image Processing, vol. 10, pp. 49-60, Jan. 2001.

[9] H. Guo, J. E. Odegard, M. Lang, R. A. Gopinath, I. W. Selesnick, and C. S. Burrus, "Wavelet based speckle reduction with application to SAR based ATD/R," in Proc. ICIP, 1994.

[10] D. L. Donoho, "Denoising by soft-thresholding," IEEE Trans. Inform. Theory, vol. 41, pp. 613-627, May 1995.

[11] A. Vidal-Pantaleoni, D. Marti, and M. Ferrando, "An adaptive multiresolution method for speckle noise reduction in synthetic aperture radar images," in Proc. IGARSS, 1999.

[12] H. Xie, L. E. Pierce, and F. T. Ulaby, "Statistical properties of logarithmically transformed speckle," IEEE Trans. Geosci. Remote Sensing, vol. 40, pp. 721-727, Mar. 2002.

[13] J. R. Sveinsson and J. A. Benediktsson, "Speckle reduction and enhancement of SAR images in the wavelet domain," in Proc. IGARSS, 1996.

[14] H. Xie, L. E. Pierce, and F. T. Ulaby, "Despeckling SAR images using a low-complexity wavelet denoising process," in Proc. IGARSS, 2002.

[15] G. P. Nason and B. W. Silverman, "The stationary wavelet transform and some statistical applications," in Wavelets and Statistics. New York: Springer-Verlag, 1995, Lecture Notes in Statistics.

[16] R. R. Coifman and D. L. Donoho, Translation Invariant De-Noising. New York: Springer-Verlag, 1995, Lecture Notes in Statistics.

[17] R. Touzi, A. Lopes, and P. Bousquet, "A statistical and geometrical edge detector for SAR images," IEEE Trans. Geosci. Remote Sensing, vol. 26, pp. 764-773, Nov. 1988.

[18] J. S. Lee, A. R. Miller, and K. W. Hoppel, "Statistics of phase difference and product magnitude of multilook processed Gaussian signals," Waves Random Media, vol. 4, July 1994.

[19] The Mathworks, "Discrete stationary wavelet transform (SWT)," The Mathworks, Natick, MA. [Online]. Available: http://www.mathworks.com.

[20] S. G. Chang and M. Vetterli, "Spatial adaptive wavelet thresholding for image denoising," in Proc. IEEE Int. Symp. Image Processing, 1997.

[21] H. Chipman, E. Kolaczyk, and R. McCulloch, "Adaptive Bayesian wavelet shrinkage," J. Amer. Statist. Assoc., vol. 92, 1997.

[22] R. A. Redner and H. F. Walker, "Mixture densities, maximum likelihood and the EM algorithm," SIAM Rev., vol. 26, no. 2, 1984.

[23] S. Foucher, J. M. Boucher, and G. B. Benie, "Maximum likelihood estimation of the number of looks in SAR images," in Proc. Int. Conf. Microwaves, Radar Wireless Communications, vol. 2, May 2000.

[24] A. F. C. Ferreira and D. Fernandes, "Speckle filter for weibull-distributed SAR images," in Proc. IGARSS, 2000.

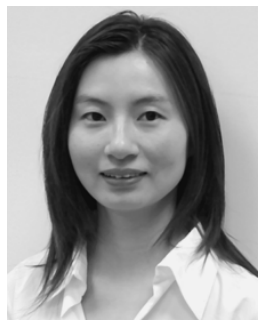

Min Dai (S'04) received the B.S. and M.S. degrees in electrical engineering from Shanghai Jiao Tong University, Shanghai, China, in 1996 and 1998, respectively. She is currently pursuing the $\mathrm{Ph} . \mathrm{D}$. degree in electrical engineering at Texas A\&M University, College Station.

Her research interests include image denoising and classification, scalable video streaming, and video traffic modeling.

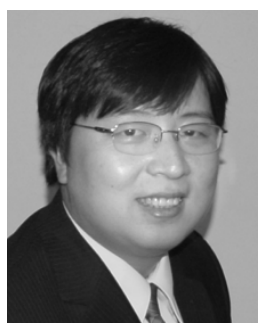

Cheng Peng received the B.S. degree in mathematics from Beijing Normal University, Beijing, China, in 1995, the M.S. degree in mathematics from Peking University, Beijing, China, in 1998, and the Ph.D. degree in electrical engineering from Texas A\&M University, College Station, in 2002.

$\mathrm{He}$ is currently with Texas Instruments Inc., Stafford, TX. His research interests include wavelet implementation, image processing, and video analysis with applications in embedded system.

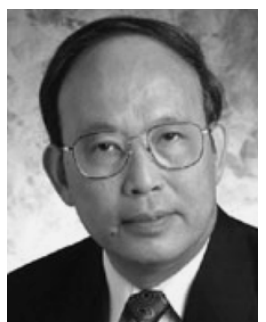

Andrew K. Chan (S'63-M'77-SM'89-F'02) received the $\mathrm{Ph} . \mathrm{D}$ degree in electrical engineering from the University of Washington, Seattle, in 1971.

He was a member of the faculty of Northern Arizona University from 1969 to 1976 . He joined the faculty of Texas A\&M University, College Station, in 1976 and is currently a Professor in the Department of Electrical Engineering. His broad research interest includes electromagnetic, acoustic, and optical wave propagation, numerical solution of nonlinear optical problems, signal and image analysis, signal detection and recognition, and wavelet analysis. His special interest is on fault detection and identification using time-frequency and time-scale techniques.

Dr. Chan is a member of Tau Beta Pi, Eta Kappa Nu Sigma Xi, and The Electromagnetic Academy.

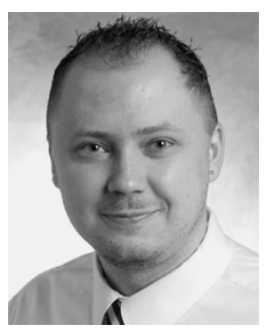

Dmitri Loguinov (S'99-M'03) received the B.S. degree (with honors) in computer science from Moscow State University, Moscow, Russia, in 1995, and the Ph.D. degree in computer science from the City University of New York, City College of New York, in 2002. Since September 2002, he has been working as Assistant Professor of computer science at Texas A\&M University, College Station. His research interests include Internet video streaming, congestion control, image and video coding, Internet traffic measurement and modeling, scalable overlay, and peer-to-peer networks, emerging QoS architectures. 